\author{
S. Hadjadj $\cdot$ R. Aubert $\cdot$ F. Fumeron $\cdot$ F. Pean $\cdot$ J. Tichet $\cdot$ \\ R. Roussel $\cdot$ M. Marre $\cdot$ SURGENE and \\ DESIR study groups
}

\title{
Increased plasma adiponectin concentrations are associated with microangiopathy in type 1 diabetic subjects
}

Received: 13 October 2004 / Accepted: 27 January 2005 / Published online: 5 May 2005

(C) Springer-Verlag 2005

\begin{abstract}
Aims/hypothesis: Insulin resistance is related to an increased risk of diabetic retinopathy and nephropathy in type 1 diabetes. Patients with insulin resistance and/or macrovascular disease have abnormally low levels of adiponectin. The aim of this study was to investigate the relationships between adiponectin and renal and retinal diabetic complications in type 1 diabetic patients. Methods: In this 6-year prospective follow-up observational study, we evaluated the severity of retinopathy at baseline and determined the incident risk of microalbuminuria in 126 normoalbuminuric patients with type 1 diabetes. Each patient was age- and sex-matched to two non-diabetic control subjects. Results: Plasma adiponectin concentrations were significantly higher in diabetic subjects than in control subjects $(p<0.0001)$. The adiponectin concentration was significantly higher in patients with severe diabetic retinopathy than in those without ( $39.1 \pm 14.0 \mathrm{vs} 29.0 \pm 13.0 \mu \mathrm{g} / \mathrm{ml}, p=0.0005$ ). The 18 patients who developed persistent microalbuminuria had higher adiponectin concentrations than the other patients $(35.8 \pm 14.5$ vs $30.6 \pm 13.7 \mu \mathrm{g} / \mathrm{ml})$. Increased adiponectin concentrations were independently associated with the occurrence of microalbuminuria $(p=0.0158)$ after adjust-
\end{abstract}

\author{
S. Hadjadj $(\bowtie)$ \\ Department of Endocrinology and Diabetology, \\ University Hospital, \\ BP 577, 86021 Poitiers Cedex, France \\ e-mail: s.hadjadj@chu-poitiers.fr \\ Tel.: +33-5-49444034 \\ Fax: $+33-5-49444006$ \\ S. Hadjadj · R. Aubert · F. Fumeron · F. Pean · R. Roussel · \\ M. Marre \\ Laboratory of Human Nutrition, \\ X Bichat Medical University INSERM U695, \\ Paris, France \\ J. Tichet \\ Inter-regional Institute for Health, \\ La Riche, France \\ R. Roussel · M. Marre \\ Department of Diabetology, Bichat Hospital, \\ Paris, France
}

ment for baseline urinary albumin concentration $(p=0.004)$, sex $(p=0.0054)$, blood pressure (NS) and metabolic control (NS). Conclusions/interpretation: The elevated adiponectin concentrations observed in subjects with microvascular disease may indicate an altered regulation of this adipocytokine in patients with complications associated with type 1 diabetes.

Keywords Adiponectin - Diabetic nephropathy ·

Diabetic retinopathy $\cdot$ Microalbuminuria $\cdot$ Microangiopathy Type 1 diabetes

Abbreviations HR: hazard ratio - eNOS: endothelial nitric oxide synthase

\section{Introduction}

Diabetic microangiopathy, specifically retinopathy and nephropathy, is a hallmark of type 1 diabetes. Diabetic retinopathy is the leading cause of sight-threatening diseases in patients of working age [1], while diabetic nephropathy is the leading cause of renal failure in France and other industrialised countries $[2,3]$. Nephropathy dramatically increases the risk of premature death [4] and cardiovascular morbidity [5] and mortality [4] associated with type 1 diabetes.

Prospective studies have recently shown that insulin resistance is significantly associated with the risk of microvascular complications in type 1 diabetic patients. Furthermore, parental insulin resistance is associated with diabetic nephropathy, which strongly suggests that insulin resistance plays a role in the development of microangiopathy in type 1 diabetes patients $[6,7]$.

Adiponectin is a hormone that is secreted by adipocytes to regulate glucose and lipid metabolism [8]. The finding that adiponectin infusion reverses insulin resistance in rodent models suggests that adiponectin plays a role in this condition [9]. Adiponectin concentrations are abnormally low in patients with insulin resistance, such as those with 
present or incident cases of type 2 diabetes [10], and in patients with macrovascular disease [11].

Based on these findings, the aim of the present study was to investigate the relationships between adiponectin and renal and retinal diabetic complications in patients with type 1 diabetes.

\section{Subjects, materials and methods}

Patient selection and follow-up We studied 126 normoalbuminuric patients, aged 25 years or over, who were among the 310 type 1 diabetic subjects included in the Survival Genetic Nephropathy (SURGENE) study, a prospective observational study [12] that was designed to analyse the determinants of diabetic nephropathy. Type 1 diabetes was diagnosed on the basis of age at disease onset $<40$ years and requirement for permanent insulin therapy within 1 year. All patients had a duration of diabetes of over 3 years and had been followed-up for over 2 years. None of the patients displayed any evidence of macrovascular disease at baseline.

The diabetic patients were compared with non-diabetic age- and sex-matched subjects who were selected from the general population to be included in the DESIR (Donnees Epidemiologiques sur le Syndrome d'Insulino-Resistance) study [13]. Each patient was compared with two non-diabetic control subjects. All patients gave written informed consent and the study protocol was approved by local ethics committees.

At baseline, retinopathy was classified by a trained ophthalmologist according to a clinical classification system, using direct fundoscopy after pupillary dilatation and/or fluorescenic angiography. Retinopathy was classified as severe (pre-proliferative retinopathy requiring laser panphotocoagulation, or past or present proliferative retinopathy identified by retinal new vessels), mild (background retinopathy, defined as micro-aneurysms and/or exudates and/or micro-hemorrhages) or absent (no evidence of retinopathy).

Patients were examined every 4-6 months for a median period of 6 years (range 2-9 years). Blood pressure was recorded using an automatic device, and $\mathrm{HbA}_{1} \mathrm{c}$ and urinary albumin concentrations were determined. Incident persistent microalbuminuria was diagnosed when the urinary albumin concentration of two or more of three consecutive random sterile samples exceeded $20 \mathrm{mg} / \mathrm{l}$, as validated previously [14].

Laboratory measurements Urinary albumin concentration was determined using a nephelometric method [15]. Fasting HDL cholesterol was determined after phosphotungstic precipitation. $\mathrm{HbA}_{1} \mathrm{c}$ was determined by HPLC (Biorad Diamat; Biorad, Ivry Sur Seine, France). Serum creatinine was determined using a modification of Jaffes's method; this was used to estimate creatinine clearance according to the Cockcroft formula [16].

Plasma adiponectin was determined in duplicate with a RIA (Linco, St Charles, MO, USA) that uses ${ }^{125}$ I-labelled murine adiponectin and multispecies adiponectin rabbit antiserum to determine plasma adiponectin levels by the double-antibody/polyethylene glycol technique. Adiponectin standards were prepared using recombinant human adiponectin. The sensitivity of this assay is $1 \mathrm{ng} / \mathrm{ml}(100 \mu \mathrm{l}$ sample size) and the intra-assay and interassay CVs are 4.4 and $9.9 \%$, respectively. The limit of linearity of the assay is $200 \mathrm{ng} / \mathrm{ml}$ (100 $\mu \mathrm{l}$ sample size). Plasma samples were diluted 1:500 in assay buffer prior to use. Samples were run in duplicate, and the means of the duplicate values were considered for statistical analyses. According to manufacturer, this RIA kit measures the trimer, hexamer, and highmolecular-weight forms, as well as some cross-reactivity to the globular domain. It does not recognize the monomer.

Statistical methods All data were stored and analysed using the STATVIEW V program (SAS Institute, Cary, NC, USA). Data are presented as mean $\pm \mathrm{SD}$, or as medians (ranges) if the distributions were skewed. Groups were compared using the chi square test for categorical variables, and parametric (if normally distributed; ANOVA or Student's $t$-test) or non-parametric tests (if not normally distributed; Mann-Whitney $U$-test) for continuous variables. The non-parametric Spearman's rank test was used to analyse the relationship between continuous variables. Multivariate logistic regression analysis was used to investigate the relationship between several candidate variables and the outcome variable (severity of retinopathy or incident microalbuminuria). An additional survival analysis using a Cox model was used to examine the contribution of adiponectin levels, with time to development of microalbuminuria as the outcome, taking potential confounding variables into account. Results are expressed as adjusted hazard ratios (HRs) with $95 \%$ confidence intervals. A $p$ value less than 0.05 was considered significant.

\section{Results}

Baseline characteristics The baseline characteristics of the type 1 diabetic patients and matched control subjects are shown in Table 1. Plasma adiponectin concentrations were significantly higher in women than in men, both in the diabetic and non-diabetic groups $(p<0.0001)$. Adiponectin concentration was correlated with age among the type 1 diabetic patients (rho $=0.238, p=0.0079$ ), but not among the control subjects (rho $=0.07$, NS). Conversely, adiponectin concentration was inversely correlated with BMI among the control subjects (rho $=-0.165, p=0.0023)$, but not among the type 1 diabetes patients $(\mathrm{rho}=-0.07, \mathrm{NS})$. We found no correlation between adiponectin and $\mathrm{HbA}_{1} \mathrm{c}$ concentrations in diabetic patients $(\mathrm{rho}=0.049$, NS). Plasma adiponectin concentration was positively correlated with HDL cholesterol concentration among the type 1 diabetic patients (rho $=0.222, p=0.0148$ ) and the non-diabetic control subjects (rho $=0.355, p<0.0001$ ). Adiponectin concentration was correlated with estimated creatinine clearance among the control subjects $(\mathrm{rho}=0.132, p=0.0409)$, but not among the type 1 diabetic patients $(\mathrm{rho}=0.006, \mathrm{NS})$. 
Table 1 Comparison of type 1 diabetic patients and control subjects

\begin{tabular}{lccl}
\hline & $\begin{array}{l}\text { Type 1 diabetic } \\
\text { patients }\end{array}$ & $\begin{array}{l}\text { Control } \\
\text { subjects }\end{array}$ & $p$ value \\
\hline Sex (men/women) & $76 / 50$ & $152 / 100$ & - \\
Age (years) & $39.1 \pm 11.7$ & $40.0 \pm 7.2$ & - \\
BMI $\left(\mathrm{kg} / \mathrm{m}^{2}\right)$ & $23.0 \pm 3.0$ & $24.2 \pm 3.6$ & 0.0014 \\
Systolic BP $(\mathrm{mmHg})$ & $125 \pm 12$ & $128 \pm 15$ & 0.042 \\
Diastolic BP $(\mathrm{mmHg})$ & $73 \pm 9$ & $79 \pm 11$ & 0.0001 \\
Serum creatinine & $76 \pm 13$ & $79 \pm 16$ & 0.09 \\
$(\mu \mathrm{mol} / \mathrm{l})$ & & & \\
Adiponectin $(\mu \mathrm{g} / \mathrm{ml})$ & & & \\
All & $31.3 \pm 13.8$ & $18.3 \pm 8.8$ & $<0.0001^{\mathrm{a}}$ \\
Men & $28.1 \pm 13.7$ & $14.0 \pm 6.3$ & $<0.0001^{\mathrm{a}}$ \\
Women & $36.2 \pm 12.6$ & $22.2 \pm 9.0$ & $<0.0001^{\mathrm{a}}$ \\
\hline
\end{tabular}

Data are means \pm SD

${ }^{\mathrm{a}} p$ value was similar with or without adjustment for BMI

The clinical and biological characteristics of the patients stratified according to diabetic retinopathy stage are summarised in Table 2. Adiponectin concentration was significantly higher in patients with severe diabetic retinopathy than in those with mild or no diabetic retinopathy ( $p=$ $0.0005)$. Logistic regression analysis showed that adiponectin concentration $(p=0.0032)$ and diabetes duration $(p=0.0001)$ were independently associated with severe retinopathy, whereas sex, BMI and systolic blood pressure were not.

Follow-up study Eighteen patients developed persistent microalbuminuria during the follow-up period (mean 6 years, range 2-9 years). The patients who developed microalbuminuria excreted more albumin in their urine and had higher adiponectin concentrations than the other patients, although these differences were statistically significant only in men (Table 3). We also found that microalbuminuric pa-

Table 2 Characteristics of type 1 diabetic patients according to diabetic retinopathy

\begin{tabular}{lllr}
\hline & $\begin{array}{l}\text { Severe } \\
\text { retinopathy }\end{array}$ & $\begin{array}{l}\text { No/mild } \\
\text { retinopathy }\end{array}$ & $p$ value \\
\hline Sex (men/women) & $18 / 11$ & $58 / 39$ & 0.8261 \\
Age (years) & $49.5 \pm 12.4$ & $35.8 \pm 9.6$ & $<0.0001$ \\
Diabetes duration & $27.9 \pm 8.4$ & $13.8 \pm 10.4$ & $<0.0001$ \\
(years) & & & \\
BMI $\left(\mathrm{kg} / \mathrm{m}^{2}\right)$ & $23.5 \pm 2.9$ & $22.8 \pm 3.0$ & 0.2801 \\
Systolic BP $(\mathrm{mmHg})$ & $130 \pm 12$ & $124 \pm 12$ & 0.0184 \\
Diastolic BP $(\mathrm{mmHg})$ & $75 \pm 8$ & $73 \pm 9$ & 0.1757 \\
HbA $\mathrm{c}(\%)$ & $8.5 \pm 1.4$ & $8.4 \pm 1.5$ & 0.6549 \\
Adiponectin $(\mu \mathrm{g} / \mathrm{ml})$ & & & \\
All & $36.2 \pm 12.6$ & $19.4 \pm 9.9$ & 0.0005 \\
Men & $36.5 \pm 14.4$ & $25.5 \pm 12.5$ & 0.0024 \\
Women & $43.3 \pm 12.9$ & $34.2 \pm 11.9$ & 0.0332 \\
\hline
\end{tabular}

Data are means \pm SD
Table 3 Characteristics of type 1 diabetic patients according to the occurrence of microalbuminuria during follow-up

\begin{tabular}{llll}
\hline & $\begin{array}{l}\text { Incident } \\
\text { microalbuminuria }\end{array}$ & $\begin{array}{l}\text { No } \\
\text { microalbuminuria }\end{array}$ & $\begin{array}{l}p \\
\text { value }\end{array}$ \\
\hline Sex (men/women) & $16 / 2$ & $60 / 48$ & 0.0074 \\
Age (years) & $40 \pm 13$ & $39 \pm 12$ & 0.6935 \\
$\begin{array}{l}\text { Diabetes duration } \\
\text { (years) }\end{array}$ & $18.3 \pm 11.1$ & $16.9 \pm 11.7$ & 0.6339 \\
BMI $\left(\mathrm{kg} / \mathrm{m}^{2}\right.$ ) & $23.1 \pm 3.6$ & $23.0 \pm 2.9$ & 0.8778 \\
$\begin{array}{l}\text { Baseline systolic BP } \\
\text { (mmHg) }\end{array}$ & $128 \pm 14$ & $125 \pm 12$ & 0.3262 \\
Mean follow-up & $133 \pm 10$ & $128 \pm 12$ & 0.1715 \\
systolic BP (mmHg) & & & \\
Baseline HbA $\mathrm{c}(\%)$ & $8.8 \pm 1.8$ & $8.3 \pm 1.0$ & 0.2706 \\
$\begin{array}{l}\text { Baseline serum } \\
\text { creatinine }(\mu \mathrm{mol} / \mathrm{l})\end{array}$ & $83 \pm 10$ & $78 \pm 16$ & 0.2174 \\
Mean follow-up & $8.4 \pm 1.0$ & $8.3 \pm 1.0$ & 0.6494 \\
HbA $(\%)$ & & & \\
Adiponectin $(\mu \mathrm{g} / \mathrm{ml})$ & & & \\
All & $35.8 \pm 14.5$ & $30.6 \pm 13.7$ & 0.1375 \\
Men & $34.6 \pm 14.8$ & $26.4 \pm 13.0$ & 0.0223 \\
Women & $45.7 \pm 6.2$ & $35.8 \pm 12.7$ & 0.2812 \\
\hline Data & & &
\end{tabular}

Data are means \pm SD

tients were more likely to be men. Logistic regression analysis showed that adiponectin $(p=0.0158)$, sex $(p=0.0054)$ and baseline urinary albumin concentration $(p=0.004)$ were independently associated with incident nephropathy, whereas diabetes duration, $\mathrm{BMI}$, mean $\mathrm{HbA}_{1} \mathrm{c}$ during follow-up and systolic blood pressure were not. A Cox model survival analysis revealed that time to development of microalbuminuria was independently and significantly determined by adiponectin concentration (adjusted HR 1.06, 95\% CI $1.02-1.10, p=0.0031$ for each increase of $1 \mu \mathrm{g} / \mathrm{ml}$ ). Other contributing variables were sex (adjusted HR 12.05, 95\% CI $2.45-62.5, p=0.0023$ for men) and urinary albumin excretion (adjusted HR 1.19, 95\% CI 1.06-1.33, $p=0.0022$ for each increase of $1 \mathrm{mg} / \mathrm{l})$. Diabetes duration $(p=0.660)$, BMI $(p=0.856), \mathrm{HbA}_{1} \mathrm{c}(p=0.305)$, and systolic $\mathrm{BP}(p=$ 0.394 ) were not associated with time to development of microalbuminuria.

A second plasma sample for the duplicate determination of adiponectin concentration was available for 38 patients (not different from the whole group) over a period of 3.2 years ( -1 to 8 years). The adiponectin concentrations measured in the duplicate samples $(-2.4 \pm 35.1 \%)$ were not different from baseline values; this was also the case in the nine patients who developed microalbuminuria $(0.9 \pm 45.1 \%)$ and the 29 who did not $(-2.9 \pm 31.9 \%)$.

\section{Discussion}

We found that the type 1 diabetic patients had higher plasma adiponectin concentrations than the sex- and agematched healthy control subjects. According to our baseline data, plasma adiponectin concentrations were indepen- 
dently higher in type 1 diabetic patients with severe retinopathy than in those with no or mild retinopathy. The results of our prospective study confirmed that elevated adiponectin was independently associated with the development of microalbuminuria in type 1 diabetic patients. This is the first report to show that an elevated concentration of adiponectin is associated with diabetic microvascular disease.

Our study was associated with certain limitations. First, it was primarily designed to study renal complications, and our data on retinal disease could not be examined using a follow-up design. Second, this study was carried out in a single centre and is of limited size, though the risk factors for diabetic retinopathy (mainly diabetes duration) and incident microalbuminuria (baseline urinary albumin excretion) were consistent with data from large-scale studies $[17,18]$.

Our results confirm recent reports that plasma adiponectin levels are higher in type 1 diabetic patients than in healthy control subjects [17-19]. In our study this difference was not due to differences in age or sex, as patients and control subjects were carefully matched. It is unlikely to be due to differences in body composition, as it persisted after adjustment for BMI.

Plasma adiponectin levels have repeatedly been shown to be lower in patients with insulin resistance or macrovascular complications than in control subjects $[8,10,11]$. We found a positive association between HDL cholesterol and plasma adiponectin levels. This reflects their association with insulin resistance in type 1 diabetic patients and in non-diabetic control subjects, and is consistent with many other observations [19]. As insulin resistance has been shown to be related to diabetic retinopathy and nephropathy in prospective $[20,21]$ and family-based studies $[6,7]$, we expected patients with severe retinopathy and incident microalbuminuria to have relatively lower levels of adiponectin. Furthermore, recent reports suggest a role for low-grade inflammation in diabetic microangiopathy, particularly that caused by IL-6 [22, 23]. As IL-6 levels are inversely correlated with adiponectin levels [24], we expected to find an association between low plasma adiponectin concentrations and microvascular disease; however, our results revealed the opposite. This finding is probably not due to differences in body composition, since subjects with severe retinopathy and those with mild or no retinopathy had similar BMIs, as did patients with and without incident microalbuminuria. It is also unlikely to be due to differences in metabolic control, since we found no correlation between $\mathrm{HbA}_{1} \mathrm{c}$ and plasma adiponectin concentrations.

Our follow-up observational study showed that higher adiponectin concentrations are associated with an increased risk of incident microvascular complications. This finding is opposite to that reported for the association between adiponectin and macrovascular complications [11]. It is therefore likely that adiponectin is regulated differently in type 1 diabetes than it is in type 2 diabetes.

The relationship between diabetic microangiopathy and adiponectin concentration has yet to be fully established, though it may be related to chronic low-grade inflammation. Unfortunately, neither highly sensitive C-reactive protein levels nor IL-6 levels were assessed in this study. Inflammation markers (possibly involved in the development of nephropathy) have previously been shown to be associated with low adiponectin concentrations in nondiabetic and type 2 diabetic subjects [25]; however, these results are at complete variance with those of the present study. Interestingly, adiponectin can stimulate angiogenesis both in vitro and in vivo [26, 27]. Thus, the increase in angiogenesis caused by the increase in adiponectin could lead to proliferative retinopathy, which would explain our results. However, we have no data to support this hypothesis in terms of the renal circulation.

The link between adiponectin and the development of microalbuminuria is currently unknown. As adiponectin levels were recently shown to be correlated with CD146 levels in healthy volunteers, it can be proposed that the increase in adiponectin promotes alterations in the endothelial junctions [28], possibly leading to leakage of albumin into the urine. Another (and not exclusive) mechanism that has been proposed is based on the fact that adiponectin induces endothelial nitric oxide production in vitro [29]. The haemodynamic hypothesis of diabetic microangiopathy emphasises the importance of increases in capillary pressure and flow as key factors for the development of complications [30]. Enhanced endothelial nitric oxide synthase (eNOS) activity has been suggested to mediate renal hyperfiltration [31]. Indeed, chronic NOS inhibition abolishes glomerular hyperfiltration [32], and the afferent arterioles of streptozotocin diabetic rats show high levels of eNOS expression [33]. This could be particularly relevant to our study, as we used the development of microalbuminuria as the study endpoint. In this regard, renal haemodynamic studies could help to determine whether this hypothesis is relevant to the association we observed between adiponectin and incident microalbuminuria. Largescale studies are needed to answer this question.

Acknowledgements We thank all the type 1 diabetes patients and control subjects who participated in this study. We thank C. Iste and V. Laneuze for their greatly appreciated secretarial assistance. We thank the French Diabetic Association (Association Française des Diabétiques), the European Union (European Rational Approach for the Genetics of Diabetic Complications [EURAGEDIC] Project QLG2-CT2001-01669), and the Diabetes and Vascular Disease Association (Association Diabète et Risque Vasculaire, Paris) for financial support. A. Edelman and Associates checked the English of the text. S. Hadjadj and R. Aubert contributed equally to this work. A list of the members of the DESIR study group is available on request.

\section{References}

1. Klein R, Klein BE, Jensen SC, Moss SE (1994) The relation of socioeconomic factors to the incidence of proliferative diabetic retinopathy and loss of vision. Ophthalmology 101:68-76

2. Pergener TV, Brancati FL, Whelton PK, Klag MJ (1994) Endstage renal disease attributable to diabetes. Ann Intern Med 121:912-918 
3. Halimi S, Zmirou D, Benhamou PY et al (1999) Huge progression of diabetes prevalence and incidence among dialysed patients in mainland France and overseas French territories. A second national survey six years apart. (UREMIDIAB 2 study). Diabetes Metab 25:507-512

4. Rossing P, Hougaard P, Borch-Johnsen K, Parving HH (1996) Predictors of mortality in insulin dependent diabetes: 10 year observational follow up study. BMJ 313:779-784

5. Tuomilheto J, Borch-Johnsen K, Molarius A et al (1998) Incidence of cardiovascular disease in type 1 (insulin-dependent) diabetic subjects with and without diabetic nephropathy in Finland. Diabetologia 41:784-790

6. Yip J, Mattock M, Sehti M, Morocutti A, Viberti G (1993) Insulin resistance in family members of insulin-dependent diabetic patients with microalbuminuria. Lancet 341:369-370

7. De Cosmo S, Bacci S, Piras GP et al (1997) High prevalence of risk factors for cardiovascular disease in parents of IDDM patients with albuminuria. Diabetologia 40:1191-1196

8. Ukkola O, Santaniemi M (2002) Adiponectin: a link between excess adiposity and associated comorbidities? J Mol Med 80: 696-702

9. Yamauchi T, Kamon J, Waki H et al (2001) The fat-derived hormone adiponectin reverses insulin resistance associated with both lipoatrophy and obesity. Nat Med 7:941-946

10. Lindsay RS, Funahashi T, Hanson RL et al (2002) Adiponectin and development of type 2 diabetes in the Pima Indian population. Lancet 360:57-58

11. Kumada M, Kihara S, Sumitsuji S et al (2003) Association of hypoadiponectinemia with coronary artery disease in men. Arterioscler Thromb Vasc Biol 23:85-89

12. Hadjadj S, Belloum R, Bouhanick B et al (2001) Prognostic value of angiotensin-I converting enzyme I/D polymorphism for nephropathy in type 1 diabetes mellitus: a prospective study. J Am Soc Nephrol 12:541-549

13. Gallois Y, Vol S, Caces E, Balkau B, and the DESIR Study Group (1996) Distribution of fasting serum insulin, measured by enzyme immunoassay, in an unselected population of 4,032 individuals, references values, according to age and sex. Diabetes Metab 22:427-431

14. Bouhanick B, Berrut G, Chameau A-M et al (1992) Predictive value of testing random urine sample to detect microalbuminuria in diabetic subjects during outpatient visit. Diabetes Metab 18:54-58

15. Marre M, Claudel JP, Ciret P, Luis N, Suarez L, Passa P (1987) Laser immunonephelometry for routine quantification of urinary albumin excretion. Clin Chem 33:209-213

16. Cockcroft DW, Gault MH (1976) Prediction of creatinine clearance from serum creatinine. Nephron 16:31-41

17. Orchard TJ, Dorman JS, Maser RE et al (1990) Prevalence in complications in IDDM by sex and duration. Pittsburgh Epidemiology of Diabetes Complications Study II. Diabetes 39: 1116-1124

18. Chaturvedi N, Bandinelli S, Mangili R, Penno G, Rottiers RE, Fuller JH (2001) Microalbuminuria in type 1 diabetes: rates, risk factors and glycemic threshold. Kidney Int 60:219-227
19. Berg AH, Combs TP, Scherer PE (2002) ACRP30/adiponectin: an adipokine regulating glucose and lipid metabolism. Trends Endocrinol Metab 13:84-89

20. Orchard TJ, Chang YF, Ferrell RE, Petro N, Ellis DE (2002) Nephropathy in type 1 diabetes: a manifestation of insulin resistance and multiple genetic susceptibilities? Further evidence from the Pittsburgh Epidemiology of Diabetes Complications Study. Kidney Int 62:963-970

21. Chaturvedi N, Sjoelie AK, Porta M et al (2001) Markers of insulin resistance are strong risk factors for retinopathy incidence in type 1 diabetes. Diabetes Care 24:284-289

22. Schram MT, Chaturvedi N, Schalkwijk C et al (2003) Vascular risk factors and markers of endothelial function as determinants of inflammatory markers in type 1 diabetes: the EURODIAB Prospective Complications Study. Diabetes Care 26:2165-2173

23. Saraheimo M, Teppo AM, Forsblom C, Fagerudd J, Groop PH (2003) Diabetic nephropathy is associated with low-grade inflammation in type 1 diabetic patients. Diabetologia 46:14021407

24. Esposito K, Pontillo A, Di Palo C et al (2003) Effect of weight loss and lifestyle changes on vascular inflammatory markers in obese women: a randomized trial. JAMA 289:1799-1804

25. Shetty GK, Economides PA, Horton ES, Mantzoros CS, Veves A (2004) Circulating adiponectin and resistin levels in relation to metabolic factors, inflammatory markers, and vascular reactivity in diabetic patients and subjects at risk for diabetes. Diabetes Care 27:2450-2457

26. Ouchi N, Kobayashi H, Kihara S et al (2003) Adiponectin stimulates angiogenesis by promoting cross-talk between AMPactivated protein kinase and Akt signaling in endothelial cells. J Biol Chem 279:1304-1309

27. Shibata R, Ouchi N, Kihara S, Sato K, Funahashi T, Walsh K (2004) Adiponectin stimulates angiogenesis in response to tissue ischemia through stimulation of AMP-activated protein kinase signaling. J Biol Chem 279:28670-28674

28. Malyszko J, Malyszko JS, Brzosko S, Wolczynski S, Mysliwiec M (2004) Adiponectin is related to CD146, a novel marker of endothelial cell activation/injury in chronic renal failure and peritoneally dialyzed patients. J Clin Endocrinol Metab 89: $4620-4627$

29. Chen H, Montagnani M, Funahashi T, Shimomura I, Quon MJ (2003) Adiponectin stimulates production of nitric oxide in vascular endothelial cells. J Biol Chem 278:45021-45026

30. Tooke JE (1995) Microvascular function in human diabetes. A physiological perspective. Diabetes 44:721-726

31. Chiarelli F, Cipollone F, Romano F et al (2000) Increased circulating nitric oxide in young patients with type 1 diabetes and persistent microalbuminuria: relation to glomerular hyperfiltration. Diabetes 49:1258-1263

32. Mattar AL, Fujihara CK, Ribeiro MO, de Nucci G, Zatz R (1996) Renal effects of acute and chronic nitric oxide inhibition in experimental diabetes. Nephron 74:136-143

33. Sugimoto H, Shikata K, Matsuda M et al (1998) Increased expression of endothelial cell nitric oxide synthase (ecNOS) in afferent and glomerular endothelial cells is involved in glomerular hyperfiltration of diabetic nephropathy. Diabetologia 41:1426-1434 